\title{
PARÂMETROS INDUSTRIAIS DA PRODUÇÃO DE DOCE DE LEITE
}

\section{Industrial parameters of doce de leite production}

\author{
Ana Carolina Paiva de Oliveira ${ }^{1}$, Sandra Maria Pinto ${ }^{2}$, Antônio Fernandes Carvalho ${ }^{3}$, \\ Pierre Schuck, Ítalo Tuler Perrone ${ }^{3 *}$, Luiz Ronaldo Abreu ${ }^{2}$
}

\begin{abstract}
RESUMO
O objetivo deste estudo foi avaliar parâmetros industriais da produção de doce de leite. Durante 37 produções em escala industrial foram determinados o rendimento, o teor de sólidos solúveis e umidade do produto, o consumo de vapor e a duração da evaporação. Correlações foram estabelecidas entre as variáveis. A equação ST $=1,02 \mathrm{SL}+1,15$, sendo $\mathrm{ST}=$ teor de sólidos totais, $\mathrm{SL}=$ teor de sólidos solúveis, descreve a relação entre ST e SL e constitui importante ferramenta para padronizar a produção e a composição do produto. A correlação mais forte estabelecida entre as variáveis ocorreu entre o tempo de produção (minutos) e o consumo de vapor $(\mathrm{kg} / \mathrm{h})$. O balanço de massa indicou uma perda média de $11,8 \%$ durante as produções. Melhorias na transferência do leite, açúcar e do produto final são indicadas como a principal solução para a redução das perdas nestas produções de doce de leite.
\end{abstract}

Palavras-chave: rendimento; sólidos solúveis; umidade; consumo de vapor.

\begin{abstract}
The aim of this study was to evaluate industrial parameters of doce de leite production. During 37 industrial productions were determined yield, soluble solids and moisture of the product, steam consumption and extent of evaporation. Mathematical correlations were stablished among variables. The equation ST = $1.02 \mathrm{SL}+1.15$, where $\mathrm{ST}=$ dry matter of the product, $\mathrm{SL}=$ soluble solids of the

1 Fundação Arthur Bernardes (FUNARBE), Viçosa, MG, Brasil.

2 Universidade Federal de Lavras (UFLA), Lavras, MG, Brasil.

3 Universidade Federal de Viçosa (UFV), Av. Peter Henry Rolfs, s/n, Campus Universitário, 36570-900, Viçosa, MG, Brasil. E-mail:italotulerperrone@gmail.com

4 UMR STLO, INRA,Agrocampus-Ouest, França.

* Autor para correspondência.
\end{abstract}


product, describes the relation between ST and SL and leads to an important tool to standardize industrial production and product composition. The highest correlation establish among variables was between extent of production (minutes) and steam consumption $\left(\mathrm{kg} / \mathrm{h}^{-1}\right)$. The mass balance indicates an average of losses of $11.8 \%$. Improvements in milk, sugar and product transfers are indicated as the main solution to reduce the losses in those doce de leite productions.

Keywords: yield; soluble solids; moisture; steam consumption.

\section{INTRODUÇÃO}

O doce de leite é um produto resultante da cocção do leite com açúcar até a concentração e caramelização desejada, apresentando elevada densidade energética (FEIHRMANN et al., 2004). É amplamente empregado como ingrediente para a elaboração de alimentos como confeitos, bolos, biscoitos, sorvetes e também consumido diretamente na alimentação (DEMIATE et al., 2001). Há uma dificuldade das empresas na padronização do doce de leite, principalmente no que diz respeito ao armazenamento do produto, para que a textura não se torne arenosa com o passar do tempo. A legislação vigente para este produto é a Portaria no 354 , Regulamento Técnico para Fixação de Identidade e Qualidade de doce de leite, de 4 de setembro de 1997 (BRASIL, 1997).

Estima-se que a produção de doce de leite representa aproximadamente $0,6 \%$ da quantidade total de produtos em um laticínio. Minas Gerais, responsável por aproximadamente $30 \%$ da produção nacional de leite, possui o maior parque industrial de laticínios do país com 34,4\% do total das empresas, detém aproximadamente $50 \%$ da produção brasileira de doce de leite, destacando-se como o principal produtor brasileiro (PERRONE et al., 2012).

O doce de leite é um produto tipicamente latino-americano, sendo produzido em larga escala em países como Brasil, Chile, e principalmente, Argentina onde o sistema de produção é contínuo, permitindo que o produto tenha características mais uniformes (MAGALHÃES, 1996). Assim como o leite condensado, leite evaporado, leite em pó e o soro em pó, o doce de leite também passa pelo processo de evaporação, que consiste na retirada de água do leite através da transferência de energia na forma de calor com vapor direto, podendo ocorrer em equipamentos que trabalham sob pressão atmosférica ou em equipamentos que trabalham sob vácuo (PERRONE et al., 2011).

A mistura do leite com a sacarose e os demais ingredientes e aditivos é denominado de calda. Esta é submetida à evaporação contínua da água por meio de transferência de energia na forma de calor de maneira indireta com vapor oriundo de caldeiras, possuindo pressão entre $100 \mathrm{kPa}$ a $600 \mathrm{kPa}$. O vapor empregado no processo é transformado em condensado de vapor, água a $85^{\circ} \mathrm{C}-90{ }^{\circ} \mathrm{C}$, e a sua quantificação possibilita determinar a massa total de vapor empregado na fabricação. O tempo de processo pode variar de 30 minutos a 4 horas, dependendo da relação entre a área de troca de energia na forma de calor e o volume de leite, bem como da pressão de vapor utilizada (PERRONE et al., 2011). Em muitas fábricas o condensado de vapor é reaproveitado como água quente para limpeza de utensílios e para a produção de vapor, acarretando economia de combustível na caldeira (PERRONE et al., 2012).

O término da produção do doce de leite ocorre pela determinação do teor de sólidos totais do produto. As dificuldades encontradas na determinação dos sólidos totais são: 
separação incompleta de água do produto; decomposição do produto com formação de água além do original; perdas das substâncias voláteis do alimento que serão computadas como peso em água; oxidação, decomposição e interação de componentes (CORDEIRO et al., 2007). Segundo Santiago et al. (2009), a relação que existe entre o teor de sólidos solúveis e o teor de sólidos totais para o doce de leite em pasta é expressa pela equação TSTD $=0,8891($ TSSD $)+8,5976$, sendo TSTD $=$ teor de sólidos totais do doce de leite em pasta, TSSD = teor de sólidos solúveis do doce de leite em pasta.

O objetivo deste estudo foi avaliar parâmetros industriais da produção de doce de leite. Durante 37 produções em escala industrial foram determinados o rendimento, o teor de sólidos solúveis e umidade do produto, o consumo de vapor e a duração da evaporação.

\section{MATERIAL E MÉTODOS}

Os dados do presente trabalho foram coletados durante a produção de 37 lotes distintos de doce de leite em escala industrial. Determinou-se em doce de leite pastoso os valores de teor de sólidos solúveis ( ${ }^{\circ} \mathrm{Brix}$ ) (no momento do ponto e no produto final após $24 \mathrm{~h}$ ), os sólidos totais do produto ( $\%$ $\mathrm{m} / \mathrm{m}$ ), o volume de leite (L) total utilizado para a produção de cada lote do produto, a massa final de doce $(\mathrm{kg})$ e a quantidade de vapor consumida por produção de cada lote (kg). O de teor de sólidos solúveis ( ${ }^{\circ}$ Brix) foi determinado através de refratômetro digital da marca Biobrix ${ }^{\circledR}$ modelo 2WAJ-D.

Para a análise de umidade das amostras utilizou-se o aparelho analisador de umidade por infravermelho IV2000, da marca Gehaka, de acordo com o manual de instruções do aparelho. O volume de leite usado na produção de cada lote foi medido antes de iniciar a produção através dos tanques de estocagem graduados, assim como foi lida a temperatura do produto.

O rendimento real do estoque (RRE) foi definido como a quantidade final de doce $(\mathrm{kg})$ verificada no setor de estoque após cada fabricação. A quantidade de vapor consumido na produção foi medida em um tanque que capta o condensado produzido durante a evaporação. O rendimento real de cada amostra de doce foi calculado usando a Equação 1.

$$
\mathrm{MDC}=\frac{(\mathrm{MLI} \% \mathrm{SLLI})+(\mathrm{MSC}+\mathrm{MR})}{(\% \mathrm{STDL})}
$$

Equação1 - Massa de doce calculada (MDC)

Sendo: $\mathrm{MDC}=$ massa de doce calculada $(\mathrm{kg})$, MLI = massa de leite integral $(\mathrm{kg}), \%$ SLLI $=$ porcentagem de sólidos láticos do leite integral $(\% \mathrm{~m} / \mathrm{m}), \mathrm{MSC}=$ massa de sacarose $(\mathrm{kg}) ; \mathrm{MR}=$ massa de redutor de acidez $(\mathrm{kg})$, $\% \mathrm{STDL}=$ porcentagem de sólidos totais do doce de leite expressos em $(\% \mathrm{~m} / \mathrm{m})$.

A partir do valor de MDC para cada lote foi calculado o rendimento empregando a Equação 2.

$$
\mathrm{RR}=\frac{\mathrm{RRE} 100}{\mathrm{MDC}}
$$

Equação 2 - Rendimento real (RR)

Sendo: $\mathrm{RR}=$ rendimento real $(\%), \mathrm{RRE}=$ rendimento real do estoque $(\mathrm{kg}), \mathrm{MDC}=$ massa de doce calculada $(\mathrm{kg})$.

Correlações e equações matemáticas empíricas derivadas de gráficos de dispersão foram estabelecidas entre as variáveis. Os dados foram submetidos à duas correlações de Pearson: uma entre consumo de vapor e o tempo de processo e outra entre consumo de 
vapor por massa de água evaporada e o tempo de processo.

\section{RESULTADOS E DISCUSSÃO}

A partir dos dados coletados obteve-se a média de rendimento real de $88,2 \% \pm 3,3 \%$, resultando em uma perda de $11,8 \% \pm 0,4 \%$ no processo de produção do doce de leite $(\mathrm{n}=37)$. A análise das etapas de fabricação indicou que são majoritariamente limitantes ao rendimento industrial as transferências de massa que ocorrem dentro da fábrica.

A passagem do leite dos tanques de recepção para os tanques de dosagem dos ingredientes e, principalmente, a transferência do doce de leite dos tachos para as máquinas de envase configuram a maior perda de massa dentro da linha do processo e consequentemente contribuem decisivamente para a redução do rendimento industrial. O setor de envase também contribuiu para a perda do rendimento devido a danos mecânicos que ocorrem durante o envase e que impossibilitam a liberação do produto, contudo esta perda foi menos relevante do que as causadas pelas transferências de massas.

Na Figura 1 é apresentada a relação entre o teor de sólidos solúveis após 24 horas de fabricação e o teor de sólidos totais do doce de leite.

A relação entre as variáveis apresentadas na Figura 1 pode ser equacionada conforme a Equação 3.

$$
\mathrm{ST}=1,02 \mathrm{SL}+1,15\left(\mathrm{R}^{2}=0,9\right)
$$

Equação 3 - Teor de sólidos totais em relação às variáveis da Figura 1.

Sendo $\mathrm{ST}=$ teor de sólidos totais $(\mathrm{m} / \mathrm{m})$ e SL $=$ teor de sólidos solúveis $\left({ }^{\circ}\right.$ Brix $)$.

A Equação 3 possui relevante aplicação prática e industrial, pois é possível prever o teor de sólidos totais do produto (o teor de umidade por diferença) a partir do teor de sólidos solúveis. O teor de umidade do doce de leite possui limite máximo estipulado pela legislação de $30 \%$ e o teor de umidade final do produto impacta diretamente no rendimento

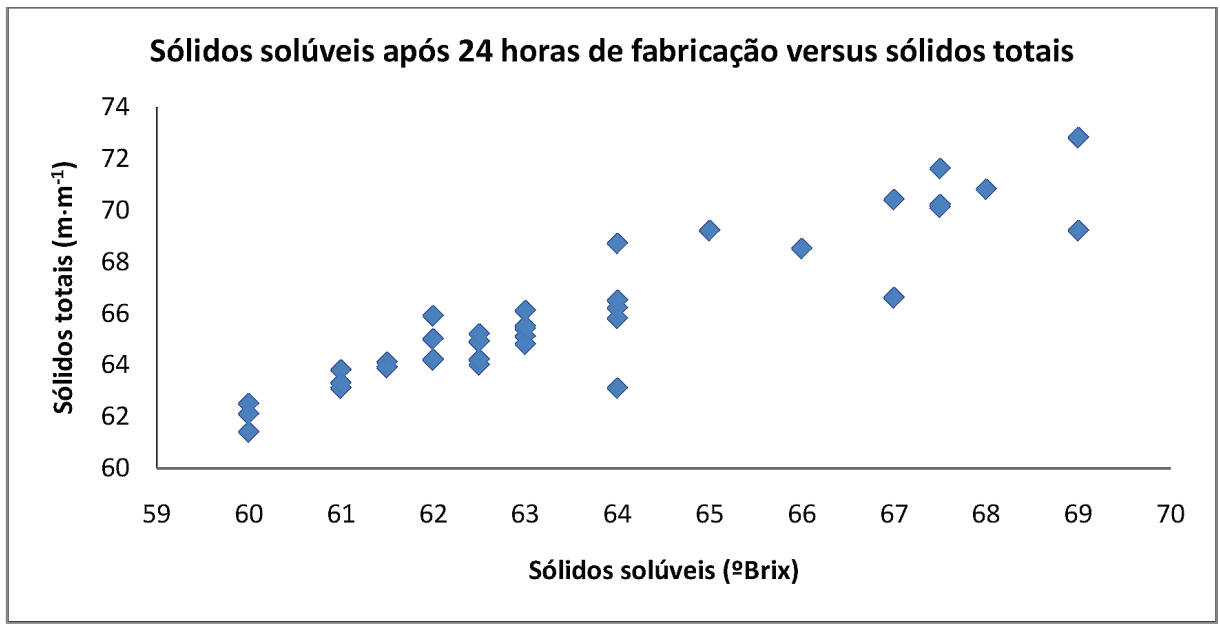

Figura 1 - Relação entre os sólidos totais do doce de leite e o teor de sólidos solúveis após 24 horas de fabricação 
da produção. Objetivando atingir o máximo de rendimento possível e mantendo o produto dentro dos requisitos legais deve-se buscar uma umidade final de 70\% (equivalente a 67,5 Brix segundo a Equação 3).

Desta forma, a possibilidade de prever o teor de umidade do produto final por meio da Equação 3 e pela quantificação do teor de sólidos solúveis (que caracteriza-se por ser uma metodologia simples e de rápida execução) configura em importante ferramenta industrial para a padronização da composição e maximização do rendimento. Esta relação ainda pode ser estabelecida entre o teor de sólidos totais do doce de leite e o teor de sólidos solúveis no momento do ponto. Esta relação é apresentada na Figura 2 e na Equação 4.

$$
\mathrm{ST}=1,084 \mathrm{SL}-2,55\left(\mathrm{R}^{2}=0,8\right)
$$

Equação 4 - Teor de sólidos totais em relação às variáveis da Figura 2

Sendo $\mathrm{ST}=$ teor de sólidos totais $(\mathrm{m} / \mathrm{m})$ e $\mathrm{SL}$ $=$ teor de sólidos solúveis $\left({ }^{\circ} \mathrm{Brix}\right)$.
A diferença entre os teores de sólidos solúveis no momento do ponto e após 24 horas de fabricação explica-se pela continuidade do processo de evaporação durante o resfriamento do doce. Desta forma, o teor de sólidos solúveis no momento do ponto sempre será de menor magnitude do que após 24 horas da fabricação. A Equação 4 deve ser aplicada no setor de produção do doce e a Equação 3 deve ser aplicada no laboratório de análises da indústria. No trabalho de Santiago et al. (2009) a relação entre o teor de sólidos totais do doce de leite e o teor de sólidos solúveis após a fabricação foi estabelecida para diferentes produtos de mercado, provenientes de formulações distintas. Desta forma, a equação estabelecida por Santiago et al. (2009) possui espectro de aplicação mais amplo, enquanto que as equações 3 e 4 estabelecidas neste estudo ajustam-se melhor à formulação e às condições industriais, principalmente por terem sido estabelecidas a partir da mensuração de 37 lotes.

Na Tabela 1 são apresentados os valores de correlação entre o consumo de vapor e o tempo de processo. Verificou-se uma forte

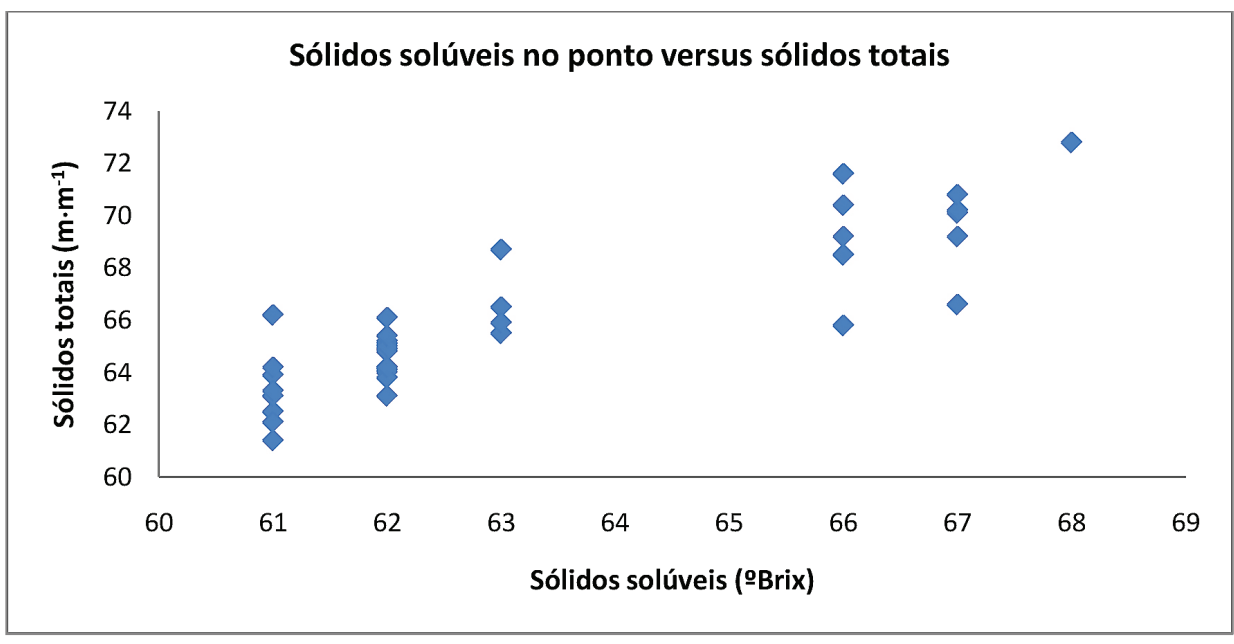

Figura 2 - Relação entre os sólidos totais do doce de leite e o teor de sólidos solúveis no momento do ponto 
correlação positiva entre consumo de vapor e o tempo de processo. A correlação entre consumo de vapor por massa de água evaporada versus tempo de processo apresentou-se positiva, porém de intensidade moderada.

A elevada correlação entre consumo de vapor $(\mathrm{kg})$ e o tempo de processo ( $\mathrm{min})$ é esperada para a evaporação em tachos abertos e deixa evidente a necessidade que as indústrias de doce de leite possuem de manter uma pressão e uma quantidade de vapor constantes e suficientes as demandas de evaporação durante todo o processamento.

\section{CONCLUSÕES}

O balanço de massa indicou uma perda média de $11,8 \%$ durante as produções. Melhorias na transferência do leite, açúcar e do produto final são indicadas como a principal solução para a redução das perdas nestas produções de doce de leite. As equações estabelecidas entre os teores de sólidos solúveis e de sólidos totais para o doce de leite possibilitam prever a umidade do produto final, configurando importante ferramenta industrial para a padronização da composição e maximização do rendimento.

\section{AGRADECIMENTOS}

Os autores agradecem ao $\mathrm{CNPq}$ e a FAPEMIG pelo suporte financeiro a projetos na área de produtos lácteos concentrados e desidratados, e, a CAPES e ao CNPq pelas bolsas de pós-doutorado e de produtividade concedidas.

\section{REFERÊNCIAS}

BRASIL. Ministério da Agricultura e do Abastecimento, Secretaria de Defesa Agropecuária, Departamento de Inspeção de Produtos de Origem Animal. Portaria n ${ }^{\circ} 354$, de 04 de setembro de 1997. Aprova o Regulamento técnico para fixação de identidade e qualidade de doce de leite. Diário Oficial da República Federativa do Brasil. Disponível em: <http://www.agricultura.gov. br>. Acesso em: 10 abr. 2016.

CORDEIRO, M. et al. Comparação dos métodos de estufa convencional e com circulação de ar forçada para desidratação de amostras de doce de leite. In: V Semana de Tecnologia de Alimentos, Ponta Grossa. Anais... Ponta Grossa: Universidade Tecnológica Federal do Paraná, 2007, v. 2, n. 1.

DEMIATE, I. M.; KONKEL, F. E.; PEDROSO, R. A. Avaliação da qualidade de amostras comerciais de doce de leite pastoso - Composição Química. Ciência e Tecnologia de Alimentos, Campinas, v. 21, n. 1, p. 108$114,2001$.

FEIHRMANN, A. C. et al. Doce de leite (revisão). Higiene Alimentar, v. 18, n. 118, p. 21-23, 2004.

Tabela 1 - Correlação de Pearson entre atributos do processamento do doce de leite

\begin{tabular}{lc}
\hline Correlação de Pearson & Valor \\
\hline Consumo de vapor $(\mathrm{kg})$ e tempo de processo $(\mathrm{min})$ & 0,99 \\
\hline Consumo de vapor por massa de água evaporada $(\mathrm{kg})$ e o tempo de processo $(\mathrm{min})$ & 0,61 \\
\hline
\end{tabular}


MAGALHÃES, F. A. R. Métodos descritivos e avaliação sensorial de doce de leite pastoso. 1996. 83p. Dissertação (mestrado em Ciência e Tecnologia de Alimentos) - Departamento de Ciência de Alimentos, Univerversidade Federal de Viçosa,Viçosa.

PERRONE, I. T. Atributos tecnológicos de controle para produção do doce de leite. Revista do Instituto de Laticínios Cândido Tostes, v. 67, n. 385, p. 42-51, 2012.
PERRONE, I. T.; STEPHANI, R.; NEVES, B. S. Doce de leite Aspectos Tecnológicos. Juiz de Fora, 2011. 186 p.

SANTIAGO, B. C. F. Correlação entre sólidos solúveis, umidade e atividade de água e determinação de amiláceos em doce de leite. In: Congresso Nacional de Laticínios, 26, 2009, Juiz de Fora. Anais... Juiz de Fora: EPAMIG, 2009. 1 CD-ROM. 